\title{
Correlation of Nuclear Morphometry with Pathologic Parameters in Ductal Carcinoma In Situ of the Breast
}

\author{
Puay Hoon Tan, F.R.C.P.A., Boon Bee Goh, Gilbert Chiang, F.R.C.P.A., Boon Huat Bay, Ph.D. \\ Department of Pathology (PHT, GC), Singapore General Hospital, Singapore; United Medical and Dental \\ School of Guy's and St. Thomas' Hospital, University of London (BBG), London, United Kingdom; and \\ Department of Anatomy, Faculty of Medicine (BHB), National University of Singapore, Singapore
}

Morphometric features of nuclear perimeter, nuclear area, feret ratio, and feret circle were studied in a series of 64 cases of ductal carcinoma in situ (DCIS) of the breast in Singapore women. The results were compared with pathologic parameters of tumor size, nuclear grade, necrosis, cell polarization, and architectural pattern. There was statistically significant correlation between nuclear perimeter and area with all the pathologic parameters, with the strongest association observed for nuclear grade $(P<.0001)$. Higher grade nuclei as assessed histologically were associated with larger nuclear area $\left(44.14 \mu \mathrm{m}^{2}\right.$ in low-grade lesions, $47.77 \mu \mathrm{m}^{2}$ in intermediate-grade lesions, and $72.05 \mu \mathrm{m}^{2}$ in highgrade lesions) and perimeter $(25.94 \mu \mathrm{m}$ in lowgrade nuclei, $27.12 \mu \mathrm{m}$ in intermediate-grade nuclei, and $33.66 \mu \mathrm{m}$ in high-grade nuclei). DCIS lesions with necrosis and absence of polarization also revealed increased nuclear area and perimeter $(P<.05)$. Comedo architecture was associated with larger nuclear area and perimeter $\left(65.97 \mu \mathrm{m}^{2}, 31.7\right.$ $\mu \mathrm{m})$ than the papillary subtype $\left(42.17 \mu \mathrm{m}^{2}, 25.29\right.$ $\mu \mathrm{m})$, with the mixed morphologic pattern disclosing intermediate values $\left(54.83 \mu \mathrm{m}^{2}, 29.43 \mu \mathrm{m}\right)$. There was direct correlation for tumor size with nuclear area and perimeter $(P<.01)$. No similar relationship was found between pathologic parameters and feret ratio or circle, indicating that nuclear roundness or lack of it did not factor as a significant component in the pathologic assessment.

KEY WORDS: Breast, DCIS, Morphometry, Pathologic parameters.

Mod Pathol 2001;14(10):937-941

Copyright (C) 2001 by The United States and Canadian Academy of Pathology, Inc.

VOL. 14, NO. 10, P. 937, 2001 Printed in the U.S.A.

Date of acceptance: May 25, 2001.

Address reprint requests to: Puay Hoon Tan, F.R.C.P.A., Department of

Pathology, Singapore General Hospital, Outram Road, Singapore 169608,

Singapore; e-mail: gpttph@sgh.gov.sg; fax: 65-2276562.
Ductal carcinoma in situ (DCIS) of the breast, generally regarded as the precursor of most invasive breast cancers, has increased dramatically in incidence, due primarily to mammographic screening (1). In Singapore, it was found to comprise $25 \%$ of screen-detected breast cancers (2), with $94 \%$ of DCIS lesions associated with radiologic calcifications (3). Because it is a heterogeneous lesion, pathological classification schemes that attempt to define its biologic potential in terms of local recurrence and progression to invasive carcinoma have evolved, and debate continues as to the best system to adopt (4).

Interobserver reproducibility studies have also been performed to define the system that allows the greatest concordance in pathologic assessment (58), with most advocating nuclear grade as the criterion that achieved the best agreement.

In this paper, we document the correlation of pathologic variables determined from routine macroscopic and microscopic assessment of DCIS lesions with morphometric nuclear features derived quantitatively from image analysis. The aims are to investigate the association between histologically assigned nuclear grade and quantitative nuclear parameters, confirm its reproducibility with an objective imaging system, and define the relationship of other pathologic criteria with the nuclear morphometric findings.

\section{MATERIALS AND METHODS}

Patients, Tumors, and Pathological Evaluation

The study population comprised 64 women diagnosed with DCIS of the breast between 1993 and 1999, obtained from the files of the Department of Pathology, Singapore General Hospital. Tissues were fixed in $10 \%$ buffered formaldehyde ( $\mathrm{pH} 7.0)$, embedded in paraffin, sectioned at $4 \mu \mathrm{m}$, and stained with hematoxylin and eosin (H\&E). The pathological features analyzed were tumor size, nuclear grade, presence or absence of necrosis or cell 
polarization, and architectural subtype. Tumor size was assessed either macroscopically or microscopically. Nuclear grade was assigned in accordance with recommendations of the 1995 NHSBSP publication (9) as low, intermediate, or high. Low-grade nuclei were monomorphic, spherical, and centrally placed with indistinct nucleoli occurring in evenly spaced cells. High-grade nuclei were large, with irregular nuclear contours, coarse chromatin, and prominent nucleoli, observed in pleomorphic, irregularly spaced cells displaying frequent mitoses. Intermediate-grade nuclei straddled in between, with mild to moderate pleomorphism that was less than that seen in high-grade nuclei; however, the cells lacked the monotony of the low-grade lesions. The presence of cell polarization was defined as a radial orientation of the cell apices toward an intercellular lumen (10). Architectural subtypes included comedo, cribriform, papillary, micropapillary, and mixed categories.

\section{Image Cytometry}

Nuclear morphometry was performed in a blinded fashion by BBG (without knowledge of the histologic assessment) on representative H\&E slides containing lesional tissue, selected for optimum histologic detail. The Kontron Electronik imaging system, comprising a light microscope (Axiophot 2, Zeiss) with a ZVS-47DE Carl Zeiss camera linked to a computer using KS400 Release 2.0 software, was used. Frozen sections were excluded because of collapse of nuclei (11). For each case, 150 ductal epithelial nuclei were randomly selected from lesional areas at a magnification of $400 \mathrm{X}$. The images were then digitized and the nuclei outlined using a mouse attached to the computer (12). Only nuclei that could be completely outlined were chosen (Fig. 1). Morphometric parameters studied were nuclear area and perimeter and feret circle and feret ratio, the latter being a ratio of minimum to maximum feret diameter, with a maximum value of 1 corresponding to a circle. Feret circle, a shape factor, is defined by the formula $\left(4 \pi\right.$ area) $/$ perimeter $^{2}$. Both feret circle and feret ratio are measures of ellipticity. Values of these morphometric parameters were generated by the computer.

\section{Statistical Analysis}

Statistical analysis was performed using the statistical software GraphPad Prism. Student's $t$ test

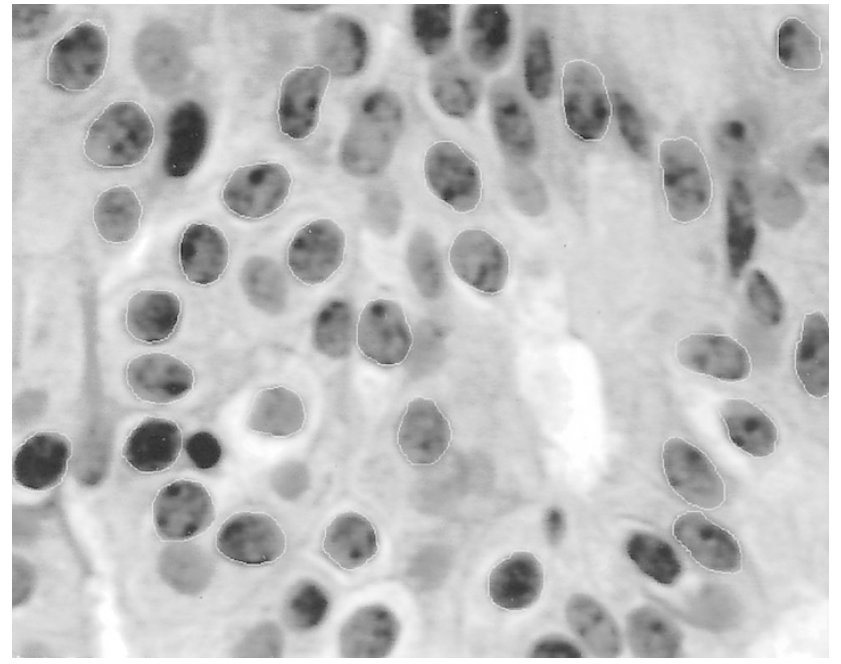

FIGURE 1. Low-grade nuclei of a ductal carcinoma in situ lesion digitally outlined using a computer mouse (hematoxylin and eosin staining, original magnification, $400 \times$ ). Cell polarization around a luminal space is noted.

was used to compare means, whereas Pearson's correlation was applied to evaluate relationships between two variables. A statistically significant result was defined as a $P$ value of $<.05$.

\section{RESULTS}

The patients were aged 33 to 82 years (mean and median, $54 \mathrm{y}$ ). Tumor size of all 64 cases ranged from 0.5 to $9 \mathrm{~cm}$, with a mean of $2.2 \mathrm{~cm}$ and a median of $1.5 \mathrm{~cm}$. Pathological findings of nuclear grade, necrosis, and cell polarization are detailed in Table 1. Examples of DCIS lesions with low, intermediate, and high nuclear grades are shown in Figure 2. Architectural classification revealed 13 (20\%) cases with comedo, 4 (6\%) with cribriform, 10 (16\%) with papillary, 2 (3\%) with micropapillary, 1 (2\%) with solid, and 34 (53\%) with mixed patterns.

Results of nuclear morphometry are summarized in Table 2. Correlation with histologic pathologic variables revealed that nuclear grade, necrosis, cell polarization, and architectural pattern were significantly associated with nuclear area and perimeter but not with feret ratio or feret circle (Table 3). Owing to insufficient numbers in the other subcategories, only comedo, papillary, and mixed architectural subtypes were analyzed statistically. There was a direct correlation of tumor size with nuclear area and perimeter $(P<.01$; Fig. 3$)$.

TABLE 1. Pathologic Features of Nuclear Grade, Necrosis, and Cell Polarization in 64 Cases of Ductal Carcinoma In Situ of Breast

\begin{tabular}{ccccccc}
\hline & Nuclear Grade & \multicolumn{3}{c}{ Necrosis } & \multicolumn{2}{c}{ Cell Polarization } \\
1 & 2 & 3 & Absent & Present & Absent & Present \\
\hline $20(31)$ & $24(38)$ & $20(31)$ & $45(70)$ & $19(30)$ & $25(39)$ & $39(61)$ \\
\hline
\end{tabular}

Data are given as number (\%). 


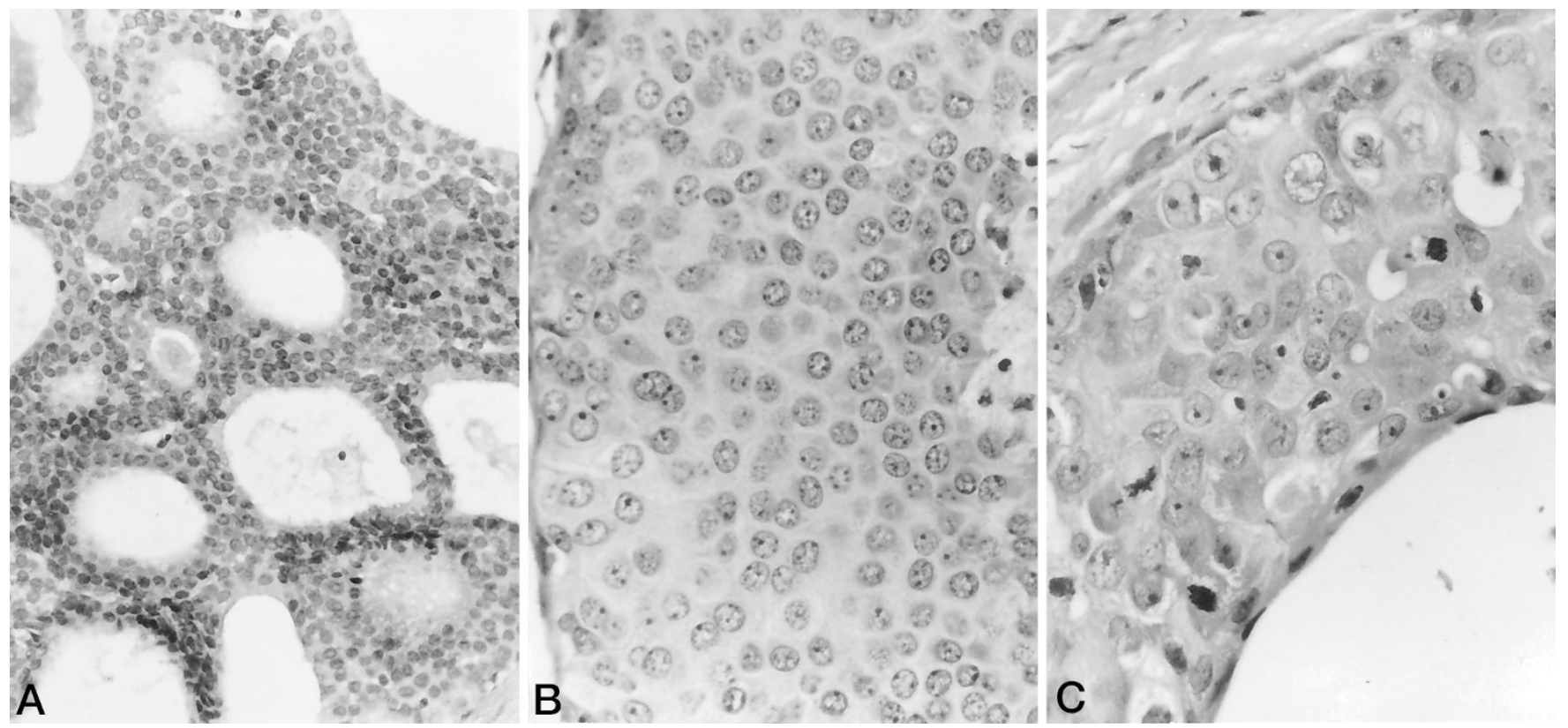

FIGURE 2. A, low-nuclear grade ductal carcinoma in situ (DCIS) reveals relatively uniform cells with spherical vesicular nuclei and indistinct nucleoli (hematoxylin and eosin staining; original magnification, $100 \times$ ). B, intermediate-nuclear grade DCIS shows moderate nuclear variation with visible nucleoli. C, high nuclear grade DCIS displays pleomorphic cells with enlarged nuclei, prominent nucleoli, and mitoses. (B and C, hematoxylin and eosin staining; original magnification, $200 \times$ ).

TABLE 2. Nuclear Morphometry in 64 Cases of Ductal Carcinoma In Situ of Breast

\begin{tabular}{lccc}
\hline \multicolumn{1}{c}{ Parameter } & Area $\left(\mu \mathrm{m}^{2}\right)$ & Perimeter $(\mu \mathrm{m})$ & Feret Ratio \\
\hline Minimum & 18.54 & 18.21 & 0.6214 \\
Maximum & 103.9 & 41.59 & 0.8033 \\
Median & 49.80 & 27.76 & 0.7734 \\
Mean & 54.29 & 28.81 & 0.7297 \\
Standard deviation & 17.87 & 4.78 & 0.7278 \\
Standard error & 2.233 & 0.5976 & 0.0389 \\
\hline
\end{tabular}

\section{DISCUSSION}

Nuclear morphometry has been shown to be of prognostic utility in invasive breast cancer (13-18). Few studies, however, have reported on its value in DCIS. Bhattacharjee et al. (19) found a cutoff difference of $20 \mu \mathrm{m}^{2}$ in mean nuclear area between diseased and normal ducts in the same section to be helpful in discriminating between epitheliosis and DCIS. Pienta et al. (20) discovered morphometric determination of nuclear pleomorphism in DCIS to be significantly increased over normal controls; and Poller et al. (21) confirmed large nuclear size in cerbB2 positive DCIS cases.

It has been established that nuclear grade is a strong predictor of recurrence after local excision (22-25) and that DCIS should be classified primarily by nuclear grade (26). Guidelines for nuclear grade assignment are based on several criteria, including the degree of pleomorphism or monotony of the lesional cell population, nuclear-cytoplasmic ratio, nucleolar prominence, and mitotic frequency (9). In addition, size comparison with normal red blood cells or unaffected duct epithelial cell nuclei is ad- vocated, with low and high nuclear grade DCIS revealing nuclear sizes 1.5 to 2.0 times and more than 2.5 times that of normal red blood cells or unaffected duct epithelial cell nuclei respectively, with intermediate-grade nuclei in between (26). It is interesting that although we graded nuclei intuitively on the subjective impression of nuclear pleomorphism without a deliberate attempt to compare sizes with red blood cells or normal duct epithelium, morphometric assessment reveals that it is the nuclear area, reflecting size, and nuclear perimeter, representing both size and shape (27), but not nuclear roundness and ellipticity (measured by feret ratio and feret circle), that are most strongly correlated. The increasing nuclear size with higher grade DCIS lesions may be explained by the accumulation of abnormal genetic material during carcinogenesis (12).

Though nuclear shape was not a significant factor in this study, it must be noted that there are inherent problems with tracing irregular nuclear contours of malignant cells for image analysis (28). Hence, the lack of association between pathologi- 


\begin{tabular}{|c|c|c|c|c|}
\hline Parameter & $\begin{array}{l}\text { Mean Nuclear Perimeter } \\
(\mu \mathrm{m})\end{array}$ & $\begin{array}{l}\text { Mean Nuclear Area } \\
\left(\mu \mathrm{m}^{2}\right)\end{array}$ & Feret Ratio & Feret Circle \\
\hline \multicolumn{5}{|l|}{ Nuclear grade } \\
\hline 1 & 25.94 & 44.14 & 0.7231 & 0.8856 \\
\hline 2 & 27.12 & 47.77 & 0.7343 & 0.8873 \\
\hline \multirow[t]{2}{*}{3} & 33.66 & 72.05 & 0.7269 & 0.8808 \\
\hline & $(P<.0001)$ & $(P<.0001)$ & $(P=.6917)$ & $(P=.7273)$ \\
\hline \multicolumn{5}{|l|}{ Necrosis } \\
\hline Absent & 26.21 & 45.13 & 0.7189 & 0.8832 \\
\hline \multirow[t]{2}{*}{ Present } & 29.99 & 58.44 & 0.7327 & 0.8856 \\
\hline & $(P=.0029)$ & $(P=.0053)$ & $(P=.1892)$ & $(P=.7482)$ \\
\hline \multicolumn{5}{|l|}{ Cell Polarization } \\
\hline Present & 27.67 & 48.76 & 0.7244 & 0.8846 \\
\hline \multirow[t]{2}{*}{ Absent } & 30.60 & 62.91 & 0.7331 & 0.8844 \\
\hline & $(P=.0153)$ & $(P=.0015)$ & $(P=.3876)$ & $(P=.9767)$ \\
\hline \multicolumn{5}{|c|}{ Architectural Pattern } \\
\hline Papillary & 25.29 & 42.17 & 0.7121 & 0.8766 \\
\hline Mixed & 29.43 & 54.83 & 0.7351 & 0.8900 \\
\hline \multirow[t]{2}{*}{ Comedo } & 31.70 & 65.97 & 0.7149 & 0.8692 \\
\hline & $(P=.0108)$ & $(P=.0075)$ & $(P=.1197)$ & $(P=.0447)$ \\
\hline
\end{tabular}

A.

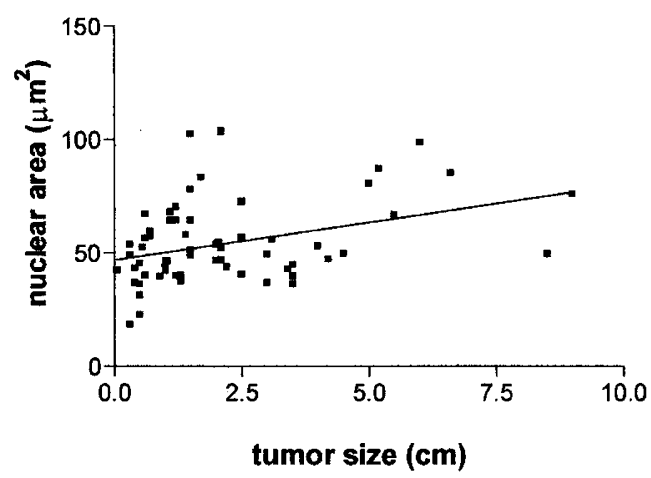

B.

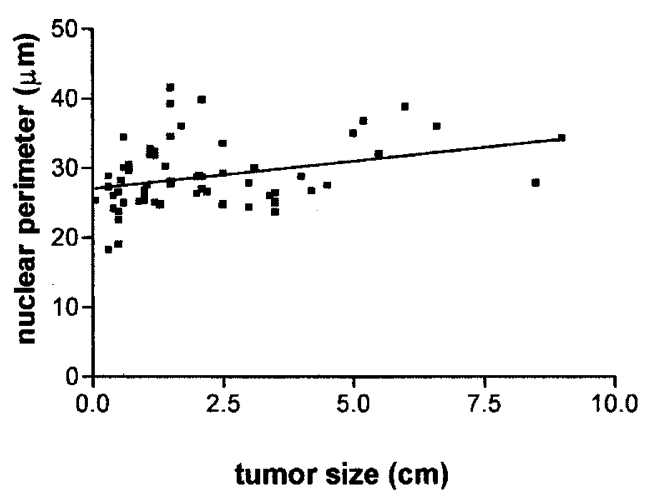

FIGURE 3. Correlation of nuclear area (A) and nuclear perimeter (B) with ductal carcinoma in situ tumor size.

cally assigned nuclear grade and the morphometric shape factors may in part be attributed to minor nuclear membrane convolutions and indentations that could have been overlooked during the digiti- zation procedure. Although the method of processing affects quantitative histopathology, nuclear shrinkage in paraffin-embedded material is less severe (29). Tumor size was also significantly associated with nuclear area and perimeter, supporting the notion that DCIS lesions containing larger nuclei tend to attain greater proportions at presentation.

Likewise, the correlation with necrosis may be partly related to larger tumor size that outstrips its blood supply and partly to greater genetic insult with induction of apoptotic cell death (30). The significant correlation between cell polarization with morphometric nuclear area and perimeter can similarly be explained on the premise that higher grade, larger nuclei lose their ability to polarize or architecturally differentiate around luminal spaces (10).

That DCIS with comedo morphology shows larger nuclei than the papillary subtype is not unexpected. It is noteworthy that lesions with a combination of patterns (mixed category) reveal mean nuclear area and perimeter that are intermediate in value. It has been recommended that nuclear grade of DCIS be based on the highest grade observed (26), despite the heterogeneity that can be encountered. Our study suggests that the mixed architectural pattern may reflect this heterogeneity in nuclear grade and perhaps lend some support to retaining the traditional classification based on morphology, which also has the additional advantage of indicating more extensive disease in the micropapillary subtype (24).

In conclusion, our study shows that the correlation between pathologic parameters of DCIS with nuclear morphometry is strongest for nuclear grade, validating the objectivity and reproducibility of nuclear grade assessment by a quantitative morphometric method. It also reveals that nuclear 
grade is correlated with nuclear enlargement than with shape factors, suggesting that nuclear size may be more useful as a criterion for grade assignment.

\section{REFERENCES}

1. Frykberg ER, Bland KI. Overview of the biology and management of ductal carcinoma in situ of the breast. Cancer 1994; 74:350-61.

2. Tan PH, Chiang GSC, Ng EH, Low SC, Ng FC. Screen detected breast cancer in an Asian population: pathological findings of the Singapore breast screening project. Breast 1999;8:120-5.

3. Tan PH, Ho JTS, Ng EH, Chiang GSC, Low SC, Ng FC, et al. Pathologic-radiologic correlations in screen-detected ductal carcinoma in situ of the breast: findings of the Singapore breast screening project. Int J Cancer (Radiat Oncol Invest) 2000;90:231-6.

4. Shoker BS, Sloane JP. DCIS grading schemes and clinical implications. Histopathology 1999;35:393-400.

5. Sloane JP, Amendoeira I, Apostolikas N, Bellocq JP, Bianchi $\mathrm{S}$, Boecker W, et al. Consistency achieved by 23 European pathologists in categorizing ductal carcinoma in situ of the breast using five classifications. European Commission Working Group on breast screening pathology. Hum Pathol 1998;29:1056-62.

6. Bethwaite P, Smith N, Delahunt B, Kenwright D. Reproducibility of new classification schemes for the pathology of ductal carcinoma in situ of the breast. J Clin Pathol 1998;51: $450-4$.

7. Sneige N, Lagios MD, Schwarting R, Colburn W, Atkinson E, Weber D, et al. Interobserver reproducibility of the Lagios nuclear grading system for ductal carcinoma in situ. Hum Pathol 1999;30:257-62.

8. Wells WA, Carney PA, Eliassen MS, Grove MR, Tosteson AN. Pathologists' agreement with experts and reproducibility of breast ductal carcinoma-in-situ classification schemes. Am J Surg Pathol 2000;24:651-9.

9. Sloane JP, Anderson TJ, Blamey RW, Brown CL, Chamberlain J, Coyne J, et al. National Coordinating Committee for Breast Screening Pathology. Pathology reporting in breast screening. Sheffield, UK: NHSBSP Publications; 1995.

10. Holland R, Peterse JL, Millis RR, Eusebi V, Faverly D, van de Vijver M, et al. Ductal carcinoma in situ: a proposal for a new classification. Semin Diagn Pathol 1994;11:167-80.

11. Boon ME, Baak JPA. Breast lesions: morphometry of cytodiagnosis. In: Baak JPA, Oort J, editors. A manual of morphometry in diagnostic pathology. New York: Springer-Verlag; 1983:96-103.

12. Huang J, Bay BH, Tan PH. Nuclear morphometry and glutathione S-transferase $\pi$ expression in breast cancer. Oncol Rep 2000;7:609-13.

13. Baak JPA, Kurver PHJ, de Snoo-Niewlaat AJE, De Graef S, Makkink B, Boon ME. Prognostic indicators in breast cancermorphometric methods. Histopathology 1982;6:327-39.

14. Baak JPA, Van Dop H, Kurver PHJ, Hermans J. The value of morphometry to classic prognosticators in breast cancer. Cancer 1985;56:374-82.
15. Aaltomaa S, Lipponen P, Eskelinen M, Kosma VM, Marin S, Alhava E, et al. The significance of nuclear morphometric variables as prognostic predictors in breast cancer. Anticancer Res 1991;11:1663-70.

16. Aaltomaa S, Lipponen P, Papinaho S, Klemi P, Kosma VM, Marin S, et al. Nuclear morphometry and DNA flow cytometry as prognostic factors in female breast cancer. Eur J Surg 1992;158:135-41.

17. Eskelinen M, Lipponen P, Papinaho S, Aaltomaa S, Kosma VM, Marin S, et al. DNA flow cytometry, nuclear morphometry, mitotic indices and steroid receptors as independent prognostic factors in female breast cancer. Int J Cancer 1992;51:555-61.

18. Kronqvist P, Kuopio T, Collan Y. Morphometric grading of invasive ductal breast cancer. I. Thresholds for nuclear grade. Br J Cancer 1998;78:800-5.

19. Bhattacharjee DK, Harris M, Faragher EB. Nuclear morphometry of epitheliosis and intraduct carcinoma of the breast. Histopathology 1985;9:511-6.

20. Pienta KJ, Coffey DS. Correlation of nuclear morphometry with progression of breast cancer. Cancer 1991;68:2012-6.

21. Poller DN, Galea M, Pearson D, Bell J, Gullick WJ, Elston CW, et al. Nuclear and flow cytometric characteristics associated with overexpression of the c-erbB-2 oncoprotein in breast carcinoma. Breast Cancer Res Treat 1991;20:3-10.

22. Badve S, A'Hern RP, Ward AM, Millis RR, Pinder SE, Ellis IO, et al. Prediction of local recurrence of ductal carcinoma in situ of the breast using five histological classifications: a comparative study with long follow-up. Hum Pathol 1998; 29:915-23.

23. Lagios MD, Margolin FR, Westdahl PR, Rose MR. Mammographically detected duct carcinoma in situ. Frequency of local recurrence following tylectomy and prognostic effect of nuclear grade on local recurrence. Cancer 1989;63:618-24.

24. Bellamy COC, McDonald C, Salter DM, Chetty U, Anderson TJ. Noninvasive ductal carcinoma of the breast: the relevance of histologic categorization. Hum Pathol 1993;24:1623.

25. Silverstein MJ. Ductal carcinoma in situ of the breast. Br Med J 1998;317:734-9.

26. Schwartz GF, Lagios MD, Carter D, Conolly J, Ellis IO, Eusebi $\mathrm{V}$, et al. Consensus conference on the classification of ductal carcinoma in situ. The consensus conference committee. Cancer 1997;80:1798-802.

27. Oort J, Baak JPA. Applications of morphometry in tumor pathology. In: Baak JPA, Oort J, editors. A manual of morphometry in diagnostic pathology. New York: Springer-Verlag; 1983:52-53.

28. Barry JD, Sharkey FE. Observer reproducibility during computer-assisted measurements of nuclear features. Hum Pathol 1985;16:225-7.

29. Ladekarl M. The influence of tissue processing on quantitative histopathology in breast cancer. J Microsc 1994;174:93100.

30. Kerr JFR, Winterford CM, Harmon BV. Apoptosis. Its significance in cancer and cancer therapy [review]. Cancer 1994; 73:2013-26. 